\title{
CONCERNING UPPER SEMI-CONTINUOUS COLLECTIONS OF CONTINUA $\left({ }^{1}\right)$
}

\author{
BY \\ R. D. ANDERSON
}

Hurewicz [1](2) and Mazurkiewicz [2] showed independently that if $M$ is any compact metric continuum, there exist a one-dimensional continuum $K$ in three-dimensional Euclidean space and an upper semi-continuous collection [3] of mutually exclusive continua filling up $K$ which with respect to its elements as points is topologically equivalent to $M$. In the case of each of these solutions the method of proof used does not lend itself readily to the solution of the principal result of this paper which is the demonstration that if $M$ is any compact continuous curve, there exist a one-dimensional continuous curve $K$ in three-dimensional Euclidean space and an upper semi-continuous collection of mutually exclusive continua filling up $K$ which with respect to its elements as points is topologically equivalent to $M$. It is known that, in the problem of Hurewicz and Mazurkiewicz, if $M$ is not a continuous curve then $K$ cannot be a continuous curve.

The principal result in this paper, Theorem II, was proposed to me as a problem by Professor R. L. Moore. I wish to express my sincere appreciation to Professor Moore for his patient and stimulating teaching and for his contagious enthusiasm for mathematical research.

Definition. A collection $Q$ of continuous curves will be said to have the $X$ property if the common part of the continua of any subcollection of $Q$ has only a finite number of components, each a continuous curve.

THEOREM I. If $M$ is any compact metric continuous curve, there exists a sequence $G_{1}, G_{2}, G_{3}, \cdots$ such that for each $i$, (1) $G_{i}$ is a finite collection of continuous curves covering $M,(2) G_{1}+G_{2}+\cdots+G_{i}$ has the $X$ property, and (3) each continuum of $G_{i}$ is of diameter less than $1 / i$.

In order to prove this theorem, four lemmas will be used. The following notation will be adopted: if $Y$ is a finite collection of continua, $N(Y)$ will be the number of elements of $Y$; if $z$ is a continuum, $D(z)$ will be the diameter of $z$.

Lemma 1. If the collection $y_{1}, y_{2}, \cdots, y_{k}, y$ has the $X$ property and the collection $y_{1}, y_{2}, \cdots, y_{k}, x$ has the $X$ property and $x$ and $y$ have a point in common then the collection $y_{1}, y_{2}, \cdots, y_{k}, y+x$ has the $X$ property.

Presented to the Society, June 19, 1948; received by the editors September 15, 1948.

(1) Presented to the Faculty of the Graduate School of the University of Texas in partial fulfillment of the requirements for the degree of Doctor of Philosophy.

(2) Numbers in brackets refer to the bibliography at the end of the paper. 
Suppose the contrary. Then the common part of the continuous curves of some subcollection $y_{n_{1}}, y_{n_{2}}, \cdots, y_{n_{i}}, y+x$ is not the sum of a finite number of continuous curves. But $y_{n_{1}} \cdot y_{n_{2}} \cdot \cdots \cdot y_{n_{i}}$ is the sum of continuous curves $m_{1}, m_{2}, \cdots, m_{e}$, and $m_{j} \cdot(y+x)$ is the sum of a finite number of continuous curves as $m_{j} \cdot y$ and $m_{j} \cdot x$ each are, and every point in $m_{j} \cdot(y+x)$ is in $m_{j} \cdot y$ or $m_{j} \cdot x$.

LEMмa 2. If the finite collection $Y$ of continuous curves has the $X$ property, and $z$, a subcontinuous curve of a continuum $N$ of $Y$, is of diameter less than $\epsilon$, then $z$ is a subset of a subcontinuous curve $z^{\prime}$ of $N$ of diameter less than $\epsilon$, such that the collection whose elements are $z^{\prime}$ and the elements of $Y$ has the $X$ property.

Suppose the contrary. Then there exists a set $Y, z$, and $\epsilon$ as in the hypothesis of the lemma for which the conclusion does not hold such that if $Y^{\prime}$, $z^{\prime}$, and $\epsilon^{\prime}$ is any set as in the hypothesis for which the conclusion does not hold, $N(Y) \leqq N\left(Y^{\prime}\right)$.

Let the continua of $Y$ be $y_{1}, y_{2}, \cdots, y_{k}[k=N(Y)]$ with $y_{k}$ corresponding to $N$ of the hypothesis. Therefore $z<y_{k}$. There exists a continuous curve $z^{\prime \prime}$ such that $z^{\prime \prime}>z, z^{\prime \prime}$ is of diameter less than $\epsilon, z^{\prime \prime}<y_{k}$, and the collection $y_{2}, y_{3}, \cdots, y_{k}, z^{\prime \prime}$ has the $X$ property. The contradiction is immediate if $y_{1}$ and $z^{\prime \prime}$ have no point in common. Suppose they do have a point in common. For each point $P$ of $y_{1} \cdot z^{\prime \prime}$ let $R$ containing $P$ be a subset of and a domain with respect to $y_{1} \cdot y_{k}$ such that $\bar{R}$ is a continuum of diameter less than $\delta=\left[\epsilon-D\left(z^{\prime \prime}\right)\right] / 2$. For each such $R$ there exists a continuous curve $K_{R}$ of diameter less than $\delta$ containing $\bar{R}$ and lying in $y_{1} \cdot y_{k}$ such that the collection $K_{R}, y_{2}, y_{3}, \cdots, y_{k-1}, C_{R}$ has the $X$ property where $C_{R}$ is the component of $y_{1} \cdot y_{k}$ containing $\bar{R}$. But some finite collection $\alpha$ of these continua $K_{R}$ covers $y_{1} \cdot z^{\prime \prime}$. The collection $y_{1}, y_{2} \cdots, y_{k}, z^{\prime \prime}+\alpha^{*}$ has the $X$ property and $z^{\prime \prime}+\alpha^{*}$ is a continuum $z^{\prime}$ yielding the desired contradiction.

Lemma 3. If $M$ is a compact continuous curve, $Y$ is a finite collection of subcontinuous curves of $M$ having the $X$ property, and $z$ is a subcontinuous curve of $M$ of diameter less than $\epsilon$, then there exists a subcontinuous curve $z^{\prime}$ of $M$ of diameter less than $\epsilon$, containing $z$, such that the collection whose elements are $z^{\prime}$ and the elements of $Y$ has the $X$ property.

This lemma follows from Lemma 2 by adding $M$ to the collection $Y$ making $z$ a subset of an element of the new collection.

LEMma 4. If the finite collection $Y$ of subcontinuous curves of the compact continuous curve $M$ has the $X$ property, and $Z$ is a finite collection of subcontinuous curves, $z_{1}, z_{2}, \cdots, z_{k}$ of $M$ each of diameter less than $\epsilon$, then there exists a collection $Z^{\prime}$ of subcontinuous curves $z_{1}^{\prime}, z_{2}^{\prime}, \cdots, z_{k}^{\prime}$ of $M$ such that for each $i, z_{i}^{\prime}>z_{i}$ and is of diameter less than $\epsilon$, and the collection $Y+Z^{\prime}$ has the $X$ property. 
Lemma 4 may be easily established with the aid of Lemma 3.

Theorem I follows as a consequence of Lemma 4.

THEOREM II. If $M$ is a compact metric continuous curve, there exists in three-dimensional Euclidean space a one-dimensional continuous curve $K$ which is filled up by an upper semi-continuous collection $G$ of mutually exclusive continua which with respect to its elements as points is topologically equivalent to $M$.

In order to prove this theorem, there will first be described a particular upper semi-continuous collection $F$ of mutually exclusive closed point sets filling up a totally disconnected closed subset $t^{\prime}$ of an interval $t$ such that with respect to its elements as points $F$ is topologically equivalent to $M$. In order to define the collection $F$ it is necessary and sufficient to define a continuous transformation $\tau$ of $t^{\prime}$ into $M$.

Let $G_{1}, G_{2}, G_{3}, \cdots$ be a sequence as in Theorem I. Let $g_{1}, g_{2}, \cdots, g_{s}$ be a finite ordering $U_{1}$ of continua of $G_{1}$ such that for each $i(i=1, \cdots, s-1), g_{i}$ has a point in common with $g_{i+1}$, and such that each continuum of $G_{1}$ occurs at least once in this ordering. Let $t$ be a straight line interval whose end points are $a$ and $b$. Let $t_{1}, t_{2}, \cdots, t_{s}$ be $s$ mutually exclusive subintervals of $t$ of equal length such that $t_{1}$ contains $a$ and $t_{s}$ contains $b$ and such that on $t, t_{i}$ separates $a$ from $t_{j}$ if and only if $1<i<j$. Call the end points of the arc $t_{i}, a_{i}$ and $b_{i}$ in the order on $t$ from $a$ to $b$. Let $\tau\left(a_{1}\right)$ be a point $P_{1}$ of $g_{1}$, let $\tau\left(b_{s}\right)$ be a point $P_{s+1}$ of $g_{s}$, and let $\tau\left(a_{i}\right)$ and $\tau\left(b_{i-1}\right)(i=2,3, \cdots, s)$ be the same point $P_{i}$ of $g_{i} \cdot g_{i-1}$. The transformation $\tau$ will be such that $\tau\left(t_{i} \cdot t^{\prime}\right)$ will be $g_{i}$. Call the initial decomposition of $M$ into $g_{1}, g_{2}, \cdots, g_{s}$ and of $t$ into $t_{1}, t_{2}, \cdots, t_{s}$ a decomposition $D_{1}$. With $D_{1}$ associate a finite set $\gamma_{1}$, of points of $M$, one in each component of $g_{j_{1}} \cdot g_{j_{1}^{\prime}}^{\prime}$ for all possible values of $j_{1}$ and $j_{1}^{\prime}$ with $j_{1} \neq j_{1}^{\prime}$ for which the common part exists.

For each continuum $g_{i}$ there exists a finite ordering $U_{1 i}$ of continuous curves $g_{i 1}, g_{i 2}, \cdots, g_{i s_{i}}$ whose sum is $g_{i}$ such that, for each $j(j=1,2, \cdots$, $\left.s_{i}-1\right), g_{i j}$ has a point in common with $g_{i j+1}$, for each $j, g_{i j}$ is a component of the common part of a continuum of $G_{2}$ and $g_{i}$ and is therefore of diameter less than $1 / 2$, and such that $g_{i 1}$ contains $P_{i}$ and $g_{i s_{i}}$ contains $P_{i+1}$. There exists a set $t_{i 1}, t_{i 2}, \cdots, t_{i s_{i}}$ of mutually exclusive subintervals of $t_{i}$ of equal length such that $t_{i 1}$ contains $a_{i}, t_{i s_{i}}$ contains $b_{i}$, and $t_{i j}$ separates $a_{i}$ from $t_{i k}$ on $t_{i}$ if and only if $1<j<k$. Call the end points of $t_{i j}, a_{i j}$ and $b_{i j}$ in the order on $t_{i}$ from $a_{i}$ to $b_{i}$. Let $\tau\left(a_{i j}\right)$ and $\tau\left(b_{i j-1}\right)$ be the same point of $g_{i j} \cdot g_{i j-1}$. We now have a second decomposition of $M$ and $t$ by means of an initial decomposition of each $g_{i}$ and $t_{i}$. Call this second decomposition $D_{2}$ and associate with $D_{2}$ a finite set $\gamma_{2}$ of points of $M$, one in each component of $g_{j_{1} k_{1}} \cdot g_{j_{1} k_{2}}$ for all possible values of the subscripts with $k_{1} \neq k_{2}$ for which the common part exists. With $D_{2}$ also associate a finite set $\delta_{1}$ of points of $M$, one in each component of $g_{j_{1} k_{1}} \cdot g_{j_{1} k_{2}} \cdot g_{j_{1}^{\prime} k_{3}}$ for all possible values of the subscripts with $k_{1} \neq k_{2}, j_{1} \neq j_{1}^{\prime}$, for which the common part exists. 
Similarly a decomposition $D_{3}$ of $M$ and $t$ may be defined by a decomposition $D_{1}$ on $D_{2}$ using $G_{3}$ as $G_{2}$ was used for $D_{2}$. With $D_{3}$ associate a finite set $\gamma_{3}$ of points of $M$, one in each component of $g_{j_{1 j_{2} k_{1}}} \cdot g_{j_{1} j_{2} k_{2}}$ for all possible values of the subscripts with $k_{1} \neq k_{2}$ for which the common part exists. With $D_{3}$ also associate a finite set $\delta_{2}$ of points of $M$, one in each component of $g_{j_{1 j_{2} k_{1}}} \cdot g_{j_{1} j_{2} k_{2}}$ - $g_{j_{1}^{\prime} j_{2} k_{3}}$ for all possible values of the subscripts with $k_{1} \neq k_{2}$ and either $j_{1} \neq j_{1}^{\prime}$ or $j_{2} \neq j_{2}^{\prime}$ for which the common part exists.

A decomposition $D_{1}$ on $D_{3}$ defines a decomposition $D_{4}$ and in this manner a sequence of decompositions may be defined, $D_{1}, D_{2}, D_{3}, \cdots$, wherein with each $D_{i}$ two finite point sets $\gamma_{i}$ and $\delta_{i-1}$ of $M$ are associated. The set $\gamma_{i}$ consists of a point of each component of $g_{j_{1} j_{2}} \cdots j_{i-1} k_{1} \cdot g_{j_{1} j_{2}} \cdots j_{i-1} k_{2}$ for all possible values of the subscripts with $k_{1} \neq k_{2}$ for which the common part exists. The set $\delta_{i-1}$ consists of a point of each component of $g_{j_{1} j_{2} \cdots j_{i-1} k_{1}} \cdot g_{j_{1} j_{2}} \cdots j_{i-1} k_{2}$ $\cdot g_{j_{1}^{\prime} j_{2}^{\prime} \cdots j_{i-1}^{\prime} k_{3}}$ for all possible values of the subscripts with $k_{1} \neq k_{2}$ and at least one $j_{e} \neq j_{e}^{\prime}$ for which the common part exists.

By means of the procedure outlined above, a continuous transformation $\tau$ of a closed totally disconnected subset $t^{\prime}$ of $t$ into $M$ is defined and there exists, defined by $\tau$, an upper semi-continuous collection $F$ of mutually exclusive closed point sets filling up $t^{\prime}$ such that, with respect to its elements as points, $F$ is topologically equivalent to $M$. If $s$ is any component of $t-t^{\prime}$, the end points of $s$ belong to the same element of $F$.

Let the interval $t$ be thrown into the interval $0 \leqq x \leqq 1, y=0, z=0$ under a reversibly continuous transformation $T$ carrying $a$ into $(0,0,0)$ and let $t$ be thrown into the interval $x=0,0 \leqq y \leqq 1, z=0$ under a reversibly continuous transformation $S$ carrying $a$ into $(0,0,0)$ such that if $P$ is any point of $t, T(P)$ and $S(P)$ are the same distance from $(0,0,0)$. Let $X^{\prime}$ be the set of $x$ coordinates of the image of $t^{\prime}$ under $T$ and $Y^{\prime}$ be the set of $y$ coordinates of the image of $t^{\prime}$ under $S$. Let $K^{\prime}$ be a continuum in three-dimensional Euclidean space consisting of the following points: the set $\mu$ of points $(x, y, z)$ such that $x$ belongs to $X^{\prime}, 0 \leqq y \leqq 1, z=0$; the set $\nu$ of points $(x, y, z)$ such that $y$ belongs to $Y^{\prime}, 0 \leqq x \leqq 1, z=1$; and the set $\omega$ of all points $P$ lying between the planes $z=0$ and $z=1$ such that for some element $Q$ of $F$ the projection of $P$ onto the $x$-axis is in $T(Q)$ and the projection of $P$ onto the $y$-axis is in $S(Q)$.

$K^{\prime}$ is not a continuous curve. There exists an upper semi-continuous collection $E^{\prime}$ of continua filling up $K^{\prime}$ such that with respect to its elements as points $E^{\prime}$ is topologically equivalent to $M$. Let $G^{\prime}$ be the particular collection defined as follows: a subcontinuum of $K^{\prime}$ belongs to $G^{\prime}$ if and only if for some element $Q$ of $F$ it consists of (1) all points $\mu_{x}$ in the set $\mu$ whose projections $x$ on the $x$-axis satisfy the property that $T^{-1}(x)$ belongs to $Q,(2)$ all points $\nu_{y}$ in the set $\nu$ whose projections $y$ on the $y$-axis satisfy the property that $S^{-1}(y)$ belongs to $Q$, and (3) all points in $K^{\prime}$ in some vertical line containing a point of $\mu_{x}$ and a point of $\nu_{y}$.

To the continuum $K^{\prime}$ will be added certain horizontal straight line seg 
ments parallel either to the $y$-axis or to the $x$-axis so that a new continuum $K$ will be defined. $K$ will be a continuous curve.

In the set $\phi$ of all the segments to be added to $K^{\prime}$ there will not be infinitely many segments all of diameter greater than the same positive number.

Let $\theta_{1}^{\prime}$ denote a countable set of segments in the plane $z=0$ parallel to the $x$-axis each having only its end points in $K^{\prime}$, such that $\theta_{1}^{\prime *}$ plus the common part of $K^{\prime}$ and the plane $z=0$ is a continuous curve. Let $\theta_{2}^{\prime}$ denote a similar set of segments in the plane $z=1$ parallel to the $y$-axis.

Let $H$ be the common part of $K^{\prime}$ and the plane $z=1 / 3$. Let $V$ denote the set of all points $P$ of $H$ such that for some point $P^{\prime}$ of $t^{\prime}, T\left(P^{\prime}\right)$ and $S\left(P^{\prime}\right)$ are the projections of $P$ onto the $x$-axis and the $y$-axis respectively. Consider the point sets $\delta_{1}, \delta_{2}, \delta_{3}, \cdots$. If $P$ is any point of $\delta_{i-1}$ there exists a finite collection $W_{P}$ such that, in order that $w$ should belong to $W_{P}$, it is necessary and sufficient that $w$ should be a set of three arcs $t_{j_{1} j_{2} \ldots j_{i-1} k_{1}}, t_{j_{1} j_{2} \cdots j_{i-1} k_{2}}$, and $t_{j_{1}^{\prime} j_{2}^{\prime} \ldots j_{i-1}^{\prime} k_{3}}$ with $k_{1} \neq k_{2}$ and for some $e, j_{e} \neq j_{e}^{\prime}$ such that $P$ is a point of a component of $g_{j_{1} j_{2}} \cdots j_{i-1} k_{1} \cdot g_{j_{1} j_{2}} \cdots j_{i-1} k_{2} \cdot g_{j_{1} j_{2}^{\prime}} \cdots j_{i-1}^{\prime} k_{3}$.

For each $i$, each point $P$ of $\delta_{i-1}$, and each element of the collection $W_{P}$, consider two points of $H$, one whose projections onto the $x$-axis and the $y$-axis are points of the images, under $T$ and $S$ respectively, of $\tau^{-1}(P)$ belonging to $t_{j_{1} j_{2}} \cdots j_{i-1} k_{1}$ and $t_{j_{1} j_{2} j^{\prime} \cdots j_{i-1}^{\prime} k_{3}}$ respectively, and another with the same projection onto the $y$-axis whose projection onto the $x$-axis belongs to $T\left(t_{j_{1} j_{2}} \cdots j_{i-1} k_{2}\right)$.

Let $s_{P w}^{\prime}$ denote the straight line segment joining these two points. Let $s_{P w}^{\prime \prime}$ denote the straight line segment obtained similarly by permuting $y$ and $S$ with $x$ and $T$ respectively in the above expressions. Let $R^{\prime}$ be the countable set of all such segments $s_{P_{w 0}}^{\prime}$ and $s_{P w_{0}}^{\prime \prime}$.

Consider the point sets $\gamma_{1}, \gamma_{2}, \gamma_{3}, \ldots$. If $Q$ is any point of $\gamma_{i}$ there exists a finite collection $W_{Q}^{\prime}$ such that in order that $w^{\prime}$ should belong to $W_{Q}^{\prime}$, it is necessary and sufficient that $w^{\prime}$ should be a set of two $\operatorname{arcs} t_{j_{1} j_{2}} \cdots j_{i-1} k_{1}$ and $t_{j_{1} j_{2}} \cdots{j_{i-1} k_{2}}$ with $k_{1} \neq k_{2}$ such that $Q$ is a point of a component of $g_{j_{1} j_{2} \cdots j_{i-1} k_{1}}$ $\cdot g_{j_{1} j_{2}} \cdots j_{i-1} k_{2}$. For each $i$, each point $Q$ of $\gamma_{i}$, and each element of the collection $W_{Q}^{\prime}$, consider a point of $H$ whose projections onto the $x$-axis and the $y$-axis are points of the images, under $T$ and $S$ respectively, of $\tau^{-1}(Q)$ belonging to $t_{j_{1} j_{2} \cdots j_{i-1} k_{1}}$ and $t_{j_{1} j_{2} \cdots j_{i-1} k_{2}}$ respectively. Let $s_{Q w^{\prime}}^{\prime}$ and $s_{Q w^{\prime}}^{\prime \prime}$ be straight line segments joining such a point with the points of $V$ having either an $x$ or a $y$ coordinate in common with it. Let $r_{Q w^{\prime}}^{\prime}$ and $r_{Q w^{\prime}}^{\prime \prime}$ denote the two straight line segments obtained similarly by permuting $y$ and $S$ with $x$ and $T$ respectively in the above expressions. Let $R^{\prime \prime}$ be the countable set of all such segments $s_{Q w^{\prime}}^{\prime}, s_{Q w^{\prime}}^{\prime \prime}, r_{Q w^{\prime}}^{\prime}$, and $r_{Q w^{\prime}}^{\prime \prime}$. Denote the elements of $R^{\prime}+R^{\prime \prime}$ by $R_{1}, R_{2}, R_{3}, \cdots$. For each $i$, add to the set $K^{\prime}$ all segments in the planes $z=[2 j-1] / 2^{i}$ for $j=1,2, \cdots, 2^{i}-1$, whose projections onto the plane $z=0$ coincide with that of $R_{i}$. Let $\theta$ denote the set of all such segments. The set $\phi$ is $\theta+\theta_{1}^{\prime}+\theta_{2}^{\prime}$.

Let $K$ be $K^{\prime}+\phi^{*}$. The continuum $K$ is a continuous curve and there exists 
an upper semi-continuous collection $G$ of mutually exclusive continua filling up $K$ which with respect to its elements as points is topologically equivalent to $M$. Each of the continua of $G$ is the sum of one continuum of $G^{\prime}$ and those segments of $\phi$ which have both end points in it.

It is clear that $K$ is connected im kleinen at every point of $K$ in the plane $z=0$ or the plane $z=1$, and at every point of the set $\phi^{*}-K^{\prime} \cdot \phi^{*}$. It suffices to show that $K$ is connected im kleinen at every point of $K^{\prime}$ for which $0<z<1$.

Let $\alpha_{1}, \alpha_{2}, \alpha_{3}, \cdots$ be a sequence of collections of parallelepipeds such that for each $i$, the collection $\alpha_{i}$ consists of all rectangular parallelepipeds with one face parallel to the plane $y=0$ and with bases in the planes $z=0$ and $z=1$ whose projection $X$ on the $x$-axis and projection $Y$ on the $y$-axis have the property that $T^{-1}(X)$ is some $t_{j_{1} j_{2}} \ldots j_{i}$ and $S^{-1}(Y)$ is some $t_{j_{1}^{\prime} j_{2}^{\prime} \cdots j_{i}^{\prime}}$ for which

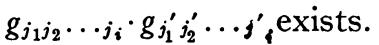

Let $\omega_{1}, \omega_{2}, \omega_{3}, \cdots$ be a sequence of collections of point sets such that in order that a point set should belong to $\omega_{i}$ it is necessary and sufficient that it should consist of all points on or in the interior of some parallelepiped of $\alpha_{i}$. Let $\beta_{1}, \beta_{2}, \beta_{3}, \cdots$ be a sequence of point sets such that for each $i, \beta_{i}$ is $\omega_{i}^{*}$.

Let $\beta$ be the closure of that subset of the continuum $K^{\prime}$ for which $0<z<1$. The point set $\beta$ is the common part of the sequence of point sets $\beta_{1}, \beta_{2}, \beta_{3}, \ldots$ and therefore $\beta+\theta^{*}$ is the common part of the sequence of point sets $\beta_{1}+\theta^{*}, \beta_{2}+\theta^{*}, \cdots$. To show that $K$ is a continuous curve it is sufficient to show that $\beta+\theta^{*}$ is a continuum, for if so the common part of any element $A$ of $\omega_{i}$ and $\beta+\theta^{*}$ is the sum of a finite number of continua, as each component of $A \cdot\left(\beta+\theta^{*}\right)$ must contain either a point or an end point of a segment of $\theta$ which also contains a point not in $A$ and there exist only a finite number of such segments in $\theta$.

To show that $\beta+\theta^{*}$ is a continuum it is sufficient to show that each $\beta_{i}+\theta^{*}$ is a continuum as the common part of a monotonic sequence of compact continua is a continuum. Because for each $i$, the ordering $U_{i}$ of continua of $M$ of the decomposition $D_{i}$ is a chain, that is, each element intersects the one which follows it in the ordering, the closure of the sum of all elements of $\omega_{i}$ which contain points of $V$ plus those elements of $\theta$ defined by use of $\gamma_{1}, \gamma_{2}, \gamma_{3}, \cdots$ is a continuum $V_{i}$.

To show that $\beta_{i}+\theta^{*}$ is a continuum it is sufficient to show that each element $A$ of $\omega_{i}$ is in the same component of $\beta_{i}+\theta^{*}$ as $V_{i}$. Let $A\left(t_{j_{1} j_{2}} \cdots j_{i}\right.$, $t_{\left.j_{1} j_{2}^{\prime} \ldots j_{i}^{\prime}\right)}$ be that element of $\omega_{i}$ whose projection on the $x$-axis is $T\left(t_{j_{1} j_{2}} \cdots j_{i}\right)$ and whose projection on the $y$ axis is $S\left(t_{j_{1}}{ }^{\prime} j_{2}^{\prime} \cdots j_{i}^{\prime}\right)$. Let $C$ be a component of $g_{j_{1} j_{2} \cdots j_{i}} \cdot g_{j_{1} j_{2}^{\prime}}^{\prime} \ldots j_{i}^{\prime}$. Consider the case for $j_{1} \neq j_{1}^{\prime}$. (If $j_{1}=j_{1}^{\prime}$, the desired result may be obtained by substituting $g_{j_{1} j_{2}} \ldots j_{e} \cdot g_{j_{1} j_{2}^{\prime}}{ }^{\prime} \ldots j_{e}^{\prime}$, with $j_{k}=j_{k}^{\prime}, k<e, j_{e} \neq j_{e}^{\prime}$, for the set $g_{j_{1}} \cdot g_{j_{1}}^{\prime}$ in what follows.) $C$ is a subset of a component $C^{\prime}$ of $g_{j_{1}} \cdot g_{j_{1}}^{\prime}$. There exist a point $P$ of $C^{\prime}$ in $\gamma_{1}$ and a segment of $\theta$ from $V_{i}$ to a point $P^{\prime}$ of $A\left(t_{j_{1}}, t_{j_{1}}{ }^{\prime}\right)$ whose image under $G$ is $P$. $P^{\prime}$ is in some $A\left(t_{j_{1} l_{2}}, t_{j_{1}}{ }^{\prime}{ }_{2}^{\prime}\right)$. Let $c_{1}, c_{2}, \cdots, c_{k}$ be a chain of components of the common parts of $C^{\prime}$ and elements of the de- 
composition $D_{2}$ of $g_{j_{1}}$ such that $c_{1}$ is the component of $g_{j_{1} l_{2}} \cdot C^{\prime}$ containing $P$, and $c_{k}$ is the component of $g_{j_{1} j_{2}} \cdot C^{\prime}$ containing $C$. Let $g_{j_{1} l_{3}}$ be an element of $D_{2}$ of $g_{j_{1}}$ containing $c_{2}$. Let $P_{1}$ be a point of $c_{1} \cdot c_{2}$ such that there is an element $g_{j_{1} m_{2}^{\prime}}{ }^{\prime}$ of $D_{2}$ of $g_{j_{1}}{ }^{\prime}$ containing $P_{1}$ for which there is a segment of $\theta$ from a point $P_{1}^{\prime}$ of $A\left(t_{j_{1} l_{2}}, t_{j_{1} m_{2}}{ }^{\prime}\right)$ to a point $P_{1}^{\prime \prime}$ of $A\left(t_{j_{1} l_{3}}, t_{j_{1} m_{2}{ }^{\prime}}\right)$ such that the image of $P_{1}^{\prime}$ under $G$ is $P_{1}$.

There exists a chain $d_{11}, d_{12}, \cdots, d_{1 h_{1}}$ of components of the common part of $c_{1}$ and elements of the decomposition $D_{2}$ of $g_{j_{1}^{\prime}}$ such that $d_{11}$ containing $P$ is in $g_{j_{1}} l_{2}^{\prime}$ and $d_{1 h_{1}}$ containing $P_{1}$ is in $g_{j_{1} m_{2}}{ }^{\prime}$. But then there exist points $P_{11}, P_{12}, \cdots, P_{1 h_{1}-1}$ such that $P_{1 i}$ is in $d_{1 i} \cdot d_{1 i+1}$ with $d_{1 i}$ in $g_{j_{1}{ }^{\prime} l_{3}{ }^{\prime}}$ and $d_{1 i+1}$ in $g_{j_{1}{ }^{\prime} l_{4}^{\prime}}$ and there is a segment of $\theta$ from a point $P_{i i}$ whose image under $G$ is $P_{1 i}$ of $A\left(t_{j_{1} l_{2}}, t_{j_{1} l_{3}}^{\prime}\right)$ to a point of $A\left(t_{j_{1} l_{2}}, t_{j_{1} l_{4}}^{\prime}\right)$. Then $A\left(t_{j_{1} l_{3}}, t_{j_{1} m_{2}{ }^{\prime}}\right)$ is in the same component of $\beta_{2}+\theta^{*}$ as $V_{2}$. But by successive reapplications of the above argument, if we use in the next stage $P_{1}$ and $t_{j_{1} l_{3}}$ as $P$ and $t_{j_{1} l_{2}}$ were used, and work along the chain $c_{1}, c_{2}, \cdots, c_{k}$, it follows that $A\left(t_{j_{1} j_{2}}, t_{j_{1} j_{2}}{ }^{\prime}\right)$ must be in the same component of $\beta_{2}+\theta^{*}$ as $V_{2}$ for it may be specified that $d_{k h_{k}}$ contains $C$ and is a subset of $g_{j_{1}{ }^{\prime} j_{2}}$. By reapplications of the above, if we use $P$ and $P_{11}$ as $P$ and $C$ were used, then $P_{11}$ and $P_{12}$ as $P$ and $C$ were used, $\cdots$, $P_{1 h_{1}-1}$ and $P_{1}$ as $P$ and $C$ were used, $\cdots$, it follows that $A\left(t_{j_{1} j_{2} j_{3}}, t_{j_{1}^{\prime} j_{2}^{\prime} j_{3}^{\prime}}\right)$ must be in the same component of $\beta_{3}+\theta^{*}$ as $V_{3}$, and finally $A\left(t_{j_{1} j_{2}} \ldots j_{i}, t_{j_{1} j_{2}}{ }^{\prime} \cdots j_{i}^{\prime}\right)$ must be in the same component of $\beta_{i}+\theta^{*}$ as $V_{i}$, as was to be shown.

The following example indicates the nature of some of the complexity of the problem of Theorem II. Let $H^{\prime}$ be a totally disconnected closed subset of the interval $0 \leqq x \leqq 1, y=0$ in the plane and let $H$ be a continuum consisting of $H^{\prime}$ and the sum of all circles with centers on the $x$-axis such that each contains two points of $H^{\prime}$ and has no point of $H^{\prime}$ in its interior. Let $\tau$ be a continuous transformation of an interval $t$ of end points $A$ and $B$ into $H$ such that there exists a point $P$ of $t$, not $A$ or $B$, such that $\tau(A P)$ is a reversibly continuous transformation, carrying the arc $A P$ into the subset of $H$ for which $y \geqq 0$, and $\tau(P B)$ is a reversibly continuous transformation, carrying the arc $P B$ into the subset of $H$ for which $y \leqq 0$.

If a two-dimensional continuum $K^{\prime}$ and a collection $G^{\prime}$ are defined as in the argument for Theorem II based on $\tau, H$, and $t$, there does not exist a continuous curve $K$ in three-dimensional Euclidean space consisting of $K^{\prime}$ and a countable number of arcs such that each intersects only one element of $G^{\prime}$, each two which intersect, intersect the same element of $G^{\prime}$, and if $\epsilon>0$, only a finite number are of diameter greater than $\epsilon$.

THEOREM III. If $M$ is a completely separable metric locally compact continuous curve, there exists a one-dimensional continuous curve $K$ in three-dimensional Euclidean space and an upper semi-continuous collection $G$ of mutually exclusive compact continua filling up $K$ which with respect to its elements as points is topologically equivalent to $M$. 
Let $D_{1}, D_{2}, D_{3}, \cdots$ be a sequence $\delta$ of compact connected domains in $M$ regarded as space such that, if $P$ is any point of $M$ and $D$ is any domain containing $P$, there exists an $i$ such that $\bar{D}_{i}$ is a subset of $D$. There exists a compact continuous curve $C_{1}$ containing $D_{1}$. There exists a finite set $\alpha_{1}$ of domains of $\delta$ covering the boundary of $C_{1}$ such that $\alpha_{1}$ includes $D_{2}$ and $\alpha_{1}^{*}$ is connected. For each domain $D$ of $\alpha_{1}$ let $C$ be a compact continuous curve containing $D$ such that the collection consisting of $C_{1}$ and the elements of the collection $\beta_{1}$ of all $C$ 's so obtained (one for each element of $\alpha_{1}$ ) has the $X$ property.

There exists a finite collection $\alpha_{2}$ of elements of $\delta$ covering the boundary of $\beta_{1}^{*}+C_{1}$ such that $\bar{\alpha}_{2}^{*}$ has no point in common with $C_{1}$ and such that $C_{1}+\beta_{1}^{*}$ $+\alpha_{2}^{*}$ is connected and covers $\sum_{1}^{3} D_{i}$. For each element $D$ of $\alpha_{2}$ there exists a compact continuous curve $C$ containing it and no point of $C_{1}$ such that if $\beta_{2}$ is the collection of all such continua (one for each element of $\alpha_{2}$ ) then the collection consisting of $C_{1}$ and the elements of $\beta_{1}$ and $\beta_{2}$ has the $X$ property.

There exists a finite collection $\alpha_{3}$ of elements of $\delta$ covering the boundary of $C_{1}+\beta_{1}^{*}+\beta_{2}^{*}$ such that the closure of no element of $\alpha_{3}$ has a point in common with $C_{1}+\beta_{1}^{*}$ and the set $C_{1}+\beta_{1}^{*}+\beta_{2}^{*}+\alpha_{3}^{*}$ is connected and covers $\sum_{1}^{4} D_{i}$. For each element $D$ of $\alpha_{3}$ there exists a compact continuous curve $C$ containing $D$ and no point of $C_{1}+\beta_{1}^{*}$ such that if $\beta_{3}$ is the collection of all such continua (one for each element of $\alpha_{3}$ ) then the collection consisting of $C_{1}$ and the elements of $\beta_{1}, \beta_{2}$, and $\beta_{3}$ has the $X$ property.

In an analogous fashion, sets $\beta_{4}, \beta_{5}, \cdots$ can be defined such that the sequence $C_{1}, \beta_{1}, \beta_{2}, \beta_{3}, \cdots$ satisfies the conditions that for each $i$, the collection consisting of $C_{1}$ and the elements of $\beta_{1}+\beta_{2}+\cdots+\beta_{i}$ is finite and has the $X$ property, for each $i>2, \beta_{i}^{*}$ contains no point of $C_{1}+\sum_{1}^{i-2} \beta_{j}^{*}$, for each $i, C_{1}+\sum_{1}^{i} \beta_{j}^{*}$ is connected, $C_{1}+\sum_{1}^{\infty} \beta_{j}^{*}$ covers $M$, and no element of $\beta_{i}$ is an element of $\beta_{i+1}$.

Let $C_{1}, C_{2}, C_{3}, \cdots$ be a sequence $\omega$ of compact continuous curves such that for each $i>1, C_{i}$ is an element of some $\beta_{k_{i}}$, for each $i$, each element of $\beta_{i}$ is a term of $\omega$, if $C_{i}$ precedes $C_{j}$ then the $\beta_{k_{i}}$ of which $C_{i}$ is an element is identical with or precedes the $\beta_{k_{j}}$ of which $C_{j}$ is an element, and for each $i$, $\sum_{i}^{i} C_{j}$ is connected. Let $B_{i}$ denote $\sum_{1}^{i} C_{j}$.

For each $i$, there exists a number $N_{i}$ such that no continuum $C_{n}$ for $n>N_{i}$ intersects any continuum $C_{j}$ for $j \leqq i$. Then by Lemma 4 of Theorem I there exists a sequence $G_{1}, G_{2}, G_{3}, \cdots$ such that $G_{1}$ is the set of continua whose elements are the terms of $\omega$, and for each $e$, (1) $G_{e}$ is a countably infinite set of compact continuous curves covering $M$ containing, for each $k$, only a finite set of continua containing points of $B_{k}$, (2) $G_{1}+G_{2}+G_{3}+\cdots+G_{e}$ has the $X$ property, (3) each continuum of $G_{e}$ for $e>1$ is of diameter less than $1 / e$, and (4) each continuum of $G_{\boldsymbol{\theta}+1}$ is a subset of some continuum of $G_{\boldsymbol{\theta}}$.

From the sequence $G_{1}, G_{2}, G_{3}, \cdots$ and by means of an argument analogous to that used for Theorem II, it may readily be shown that there exists a se- 
quence $K_{B_{1}}^{\prime}, K_{B_{2}}^{\prime}, K_{B_{3}}^{\prime}, \cdots$ and a sequence $K_{B_{1}}, K_{B_{2}}, K_{B_{3}}, \cdots$ such that (1) for each $i, K_{B_{i}}^{\prime}$ is a compact continuum in three-dimensional Euclidean space, $K_{B_{i}}$ is a one-dimensional compact continuous curve in three-dimensional Euclidean space which contains $K_{B_{i}}^{\prime}$, there exist an upper semi-continuous collection $G_{B_{i}}$ of mutually exclusive continua filling up $K_{B_{i}}$ and an upper semicontinuous collection $G_{B_{i}}^{\prime}$ of mutually exclusive continua filling up $K_{B_{i}}^{\prime}$ such that each collection with respect to its elements as points is topologically equivalent to $B_{i}$ and the continua $K_{B_{i}}$ and $K_{B_{i}}^{\prime}$ and collections $G_{B_{i}}$ and $G_{B_{i}}^{\prime}$ are obtained by a method analagous to that used in the proof of Theorem II, (2) for each $i, K_{B_{i+1}}$ contains $K_{B_{i}}$, every element of $G_{B_{i}}$ is a subset of an element of $G_{B_{i+1}}$, and no two elements of $G_{B_{i}}$ are in the same element of $G_{B_{i+1}}$, (3) for each $i$, every point of $K_{B_{i}}^{\prime}$ in the plane $z=1 / 2$ whose projection on the $x$-axis lies in the interval $\langle 2 j-1,2 j\rangle$ and whose projection on the $y$-axis lies in the interval $\langle 2 j-1,2 j\rangle$ is in the inverse image under the transformation $T_{B_{i}}$ defined by $G_{B_{i}}^{\prime}$ of $C_{j}$, (4) for each $i$, that part of $K_{B_{i}}$ for which $x \leqq 2 i-2$ and $y \leqq 2 i-2$ is identical with $K_{B_{i-1}}$, and (5) for each $i$, no point of $K_{B_{i}}$ in the plane $z=0$ has an $x$ projection lying in a segment of the $x$-axis $\langle 2 j, 2 j+1\rangle$, $j=1,2,3, \cdots$, and no point of $K_{B_{i}}$ in the plane $z=1$ a $y$ projection lying in a segment of the $y$-axis $\langle 2 j, 2 j+1\rangle, j=1,2,3, \cdots$. This last condition is important with reference to the adding of segments in the plane $z=0$ for, in some cases, under the other conditions of this paragraph, the end points of a segment $\langle 2 j, 2 j+1\rangle$ in the $x$-axis cannot belong to the same element of $G_{B_{i}}$.

The continuous curve $K=K_{B_{1}}+K_{B_{2}}+K_{B_{3}}+\cdots$ contains a continuous curve satisfying the conditions of the theorem. For each integer $i$, there exists a number $j_{i}$ such that there is no vertical segment in $K$ either whose $x$ coordinate is less than or equal to $i$ and whose $y$ coordinate is greater than $j_{i}$ or whose $y$ coordinate is less than or equal to $i$ and whose $x$ coordinate is greater than $j_{i}$. For each even integer $i$, delete from $K$ every point in the plane $z=0$ whose $x$ coordinate is less than or equal to $i$ and whose $y$ coordinate is greater than $j_{i}$, and in the plane $z=1$ delete every point whose $y$ coordinate is less than or equal to $i$ and whose $x$ coordinate is greater than $j_{i}$.

The resulting continuum is a continuous curve and there does exist an upper semi-continuous collection of compact continua filling it up which with respect to its elements as points is topologically equivalent to $M$.

The following theorem can be proved by an argument similar to but much shorter than the argument for Theorem III.

Theorem IV. If $M$ is a completely separable metric locally compact continuum, there exist a one-dimensional continuum $K$ in three-dimensional Euclidean space and an upper semi-continuous collection $G$ of mutually exclusive compact continua filling up $K$ which with respect to its elements as points is topologically equivalent to $M$.

This theorem can also be shown as a consequence of Theorem III. 


\section{BIBLIOGRAPHY}

1. Witold Hurewicz, Über oberhalb-stetige Zerlegungen von Punktmengen in Kontinua, Fund. Math. vol. 15 (1930) pp. 57-60.

2. Stefan Mazurkiewicz, Sur les images continues des continus, Comptes Rendus du Premier Congrès des Mathematiciens des Pays Slaves, Warsaw, 1929-1930, pp. 66-67.

3. - Sur les images continues des continus, Fund. Math. vol. 17 (1931) p. 330.

4. R. L. Moore, Foundations of point set theory, Amer. Math. Soc. Colloquium Publications, vol. 13, 1932.

THE UNIVERSiTY OF TEXAS, Austin, Tex. 